\title{
A morphological approach for distinguishing texture and individual features in images
}

\author{
Igor Zingman ${ }^{\mathrm{a}, * *}$, Dietmar Saupe ${ }^{\mathrm{a}}$, Karsten Lambers ${ }^{\mathrm{b}}$ \\ ${ }^{a}$ Department of Computer and Information Science, University of Konstanz, Germany \\ ${ }^{b}$ Institute of Archaeology, Heritage Sciences and Art History, University of Bamberg, Germany
}

ABSTRACT

We present a morphological texture contrast (MTC) operator that allows detection of textural and non-texture regions in images. We show that in contrast to other approaches, the MTC discriminates between texture details and isolated features and does not extend borders of texture regions. A comparison with other methods used for texture detection is provided. Using the ideas underlying the MTC operator, we develop a complementary operator called morphological feature contrast (MFC) that allows extraction of isolated features while not being confused by texture details. We illustrate an application of the MFC operator to extraction of isolated objects such as individual trees or buildings that should be distinguished from forests or urban centers. We also propose an MFC based detector of isolated linear features and compare it with an alternative approach used for detection of edges and lines in cluttered scenes. We furthermore derive an extended version of the MFC that can be directly applied to vector-valued images.

\section{Introduction}

This paper ${ }^{1}$ focuses on the problem of distinguishing isolated features from features that are part of a texture. We will refer to the latter features as texture details. Isolated features, also called individual features, are, for example, isolated ridges (bars) or small blobs in images (peaks in the 1D case). This problem may occur when one wants to detect texture regions, and at the same time distinguish them from isolated features that should not be assigned to a texture class. A dual problem occurs when it is necessary to detect isolated features avoiding detection of parts of neighboring or background texture even if texture details are similar to features of interest. For example, one may want to detect individual trees distinguishing them from trees of a forest. Here we consider both problems, namely detection of texture and of individual features.

Although a large variety of texture classification methods has been developed, much less attention has been given to the apparently simpler problem of texture detection that discriminates between texture (of any type) and non-texture regions. This is not a simple task if accurate localization is required and if texture must be distinguished from individual features.

In Dinstein et al. (1984) it was proposed to use the difference between maximal and minimal intensities (MaxMin diff.) in a pixel neighborhood for a fast segmentation of an image into textured and non-texture regions. A standard deviation $(\mathrm{StD})$ is frequently used as a measure of texture that describes its smoothness (Gonzalez and Woods, 2002). In Ojala et al. (2002), where the Local Binary Patterns (LBP) approach was

\footnotetext{
${ }^{* *}$ Corresponding author:

e-mail: igor.zingman@uni-konstanz.de (Igor Zingman)

${ }^{1}$ This is a minor revision of the version to be published in Pattern Recognition Letters. DOI: 10.1016/j.patrec.2014.03.019
}

developed, the authors also suggested to incorporate a variance based descriptor for texture classification purposes. While the LBP descriptor is related to inherent texture properties, a complementary variance based descriptor measures texture contrast. The amplitude modulation function (AMF), derived from the amplitude-modulation frequency-modulation model (Kokkinos et al., 2009), can locally capture texture contrast. Although each of the texture contrast descriptors mentioned above can be used to discriminate between texture regions and non-texture areas, also called smooth areas in this paper, they cannot distinguish individual features from texture details.

Several descriptors were suggested to approach this problem. In Verbeek et al. (1988) the difference between closing and opening, called texture range (TER), was suggested to distinguish individual step and ramp edges from texture edges. The TER operator, however, cannot distinguish isolated features, such as ridges and blobs, from texture details of comparable size. Recently, in Pesaresi et al. (2008) the PanTex index was developed to detect settlements in panchromatic satellite imagery. The operator is able to distinguish texture areas from individual linear features such as roads or borders between homogenous cultivated fields in satellite images. The PanTex index is defined as a minimal contrast among contrast measures derived from the gray-level co-occurrence matrixes (GLCM) (Haralick et al., 1973), computed for different orientations of displacement vectors. The PanTex method, however, does not distinguish other individual features, such as isolated peaks or small isotropic blobs, from texture. The component count (CC) method (Bergman et al., 2008) is based on the product of two measures computed in small image blocks. The first one is the sum of the number of connected components (component count) in the background and the foreground obtained by simple binarization of image blocks. The second measure is the difference between average intensities in the background 
and the foreground. This descriptor is supposed to discriminate blocks covering texture and individual step edges at the borders between homogenous regions. A similar idea of counting the number of local extrema (texture primitives) for detection of texture regions was proposed earlier in Karu et al. (1996). Since this method does not take into account contrast of texture primitives, it can be very sensitive to noise.

Another disadvantage that all the above texture descriptors, excluding the TER, have in common, is that they extend or blur the borders of texture regions, preventing accurate localization of texture borders. Recently, we introduced a morphological texture contrast (MTC) descriptor that does not suffer from the above disadvantages (Zingman et al., 2012). This operator, reviewed in Sec. 2, measures the difference between upper and lower texture envelopes estimated by means of alternating morphological filters (Serra and Vincent, 1992; Soille, 2003). Its qualitative performance was illustrated in Zingman et al. (2012), where only few remotely sensed images were used and no quantitative comparison was provided. In Sec. 3 we provide a quantitative comparison using artificially created images and a qualitative comparison using a set of standard test images. In this paper, we also define an alternative texture descriptor that is computed as difference between alternating sequential filters (ASF diff.) and compare it to the MTC.

As we stated in the beginning of this section, the dual problem to the problem of texture detection is detecting individual features while distinguishing them from texture details. This problem has mainly been treated in the context of edge detection capable of discarding texture surroundings. For example, recently in Grigorescu et al. (2003) a surround inhibition mechanism was introduced to improve edge detection at region boundaries. Dubuc and Zucker (2001) proposed a normal complexity measure that is able to separate isolated curves and isolated edges from texture in binary images. The paper provides an original theoretical framework, but it seems to be computationally very expensive.

In Sec. 4 we show how the ideas underlying the MTC operator lead to a Morphological Feature Contrast (MFC) operator that aims at the detection of small isolated objects, rather than edges, in textured background. We illustrate the potential of the MFC operator on gray-scale images and derive its extension to vector-valued images. Additionally, we show how the MFC operator can be incorporated into a scheme for extracting isolated linear features. We show the advantages of this scheme over the approach for the detection of contours with texture background suppression introduced in Grigorescu et al. (2003). A preliminary short version of our work was recently presented in Zingman et al. (2013b).

\section{Detection of texture regions: The Morphological Texture Contrast operator and the ASF difference}

Below, we define the morphological texture contrast (MTC) transformation $\psi_{\mathrm{MTC}}(f)$ that we recently introduced in Zingman et al. (2012) for distinguishing texture regions (such as forests, urban areas and rocky mountains) in satellite images from smooth areas, which may also contain individual struc- tures that should not to be assigned to texture ${ }^{2}$. Qualitatively, MTC's response is summarized in the first row of Table 1.

The MTC is based on alternating morphological filters, $\gamma_{r} \varphi_{r}$ and $\varphi_{r} \gamma_{r}$, which are closing $\varphi$ followed by opening $\gamma$ and opening followed by closing, respectively. $r$ denotes the size of the structuring element (SE). Alternating filters are usually employed for noise filtering. We use them to estimate texture envelopes. The difference between upper and lower envelopes defines a measure of texture contrast, which can serve as an indicator of the presence of texture

$$
\psi_{\mathrm{MTC}}(f)=\left|\gamma_{r} \varphi_{r}(f)-\varphi_{r} \gamma_{r}(f)\right|^{+},
$$

where the argument $f$ denotes a 1D signal or a 2D gray-scale image, and $|\cdot|^{+}$is defined as

$$
|v|^{+} \triangleq \begin{cases}v, & v>0 \\ 0, & \text { otherwise } .\end{cases}
$$

A remarkable property of these envelopes is that they coincide at individual features, thereby yielding low response at individual features even if they are of high contrast (see an example in Fig. 1). Since in the 2D case, $\varphi_{r} \gamma_{r}$ and $\gamma_{r} \varphi_{r}$ are not ordered (Serra and Vincent, 1992; Soille, 2003), a lower envelope might be above an upper envelope. However, Proposition 3 below shows that regions where this happens are small in the sense that an erosion with a structuring element of size $r$ completely removes these regions. In the following discussion we will show that $r$ defines the minimal size of texture regions to be detected (see Eq. (9)). Therefore, the regions where $\gamma_{r} \varphi_{r}<\varphi_{r} \gamma_{r}$ are small enough to be considered as non-texture regions. They are correspondingly removed by the $|.|^{+}$operator in the definition of $\psi_{M T C}$ above.

Let us denote morphological erosion of a set or a function by $\varepsilon$. Large letters will denote sets. Structuring elements are identical for all morphological operators in the following propositions.

Proposition 1. The following inequality holds: $\varepsilon \gamma \varphi \geq \varepsilon \varphi \gamma$.

Proof. We have $\varepsilon \varphi \geq \varepsilon \varphi \gamma$ due to the increasing property of closing and erosion, and antiextensivity of opening. Proposition 1 follows directly from the last inequality and due to $\varepsilon \gamma=\varepsilon$

Proposition 2. Given the ordering condition $g_{1}(x)<g_{2}(x), x \in$ $D$ the following inequality holds: $\left[\varepsilon\left(g_{1}\right)\right](y)<\left[\varepsilon\left(g_{2}\right)\right](y), y \in$ $\varepsilon(D)$.

Proof. Let us denote by $B_{y}$ a structuring element shifted to position $y$. For $y \in \varepsilon(D)$ we have $\left[\varepsilon\left(g_{1}\right)\right](y)=\min _{x \in B_{y} \subseteq D} g_{1}(x)<$ $\min _{x \in B_{y} \subseteq D} g_{2}(x)=\left[\varepsilon\left(g_{2}\right)\right](y)$, where the inequality follows from the given ordering condition.

\footnotetext{
${ }^{2}$ This work was motivated by an archaeological project (Lambers and Zingman, 2012, in press) that targets detection of individual architectural remains located in open grassland areas.
} 
Proposition 3. Given the set $X=\{x: \gamma \varphi<\varphi \gamma\}$, the set $Y=$ $\{y: y \in \varepsilon(X)\}$ is an empty set.

Proof. From the construction of the sets $X, Y$ and from Proposition 2 with $g_{1}(x)=[\gamma \varphi(f)](x)$ and $g_{2}(x)=[\varphi \gamma(f)](x)$, for $x \in X$, it follows that $[\varepsilon \gamma \varphi(f)](y)<[\varepsilon \varphi \gamma(f)](y)$, for $y \in Y$. Since the last inequality contradicts Proposition 1 we conclude that $Y$ is empty.

The results of applying the MTC to an artificial 1D signal and to a remotely sensed image ${ }^{3}$ of a forested area are shown in Fig. 1(right) and Figs. 2(b, c), respectively. Note that individual trees in Fig. 2, and individual peaks as well as step edges (front and back of the wide pulse) in Fig. 1 were suppressed. Throughout this paper we use square SEs, where the size refers to its side length. The size $r$ of the SE of $\psi_{\text {MTC }}$ should be chosen to be larger than the maximal distance between details in textured regions. Features that stand apart from texture details farther than $r$ are treated as individual features and are suppressed correspondingly. In general, we can use different sizes $r_{1} \neq r_{2}$ for SEs of $\psi_{\mathrm{MTC}}$,

$$
\psi_{\mathrm{MTC}}(f)=\left|\gamma_{r_{2}} \varphi_{r_{1}}(f)-\varphi_{r_{2}} \gamma_{r_{1}}(f)\right|^{+} .
$$

The size of the SEs $r_{1}$ and $r_{2}$ should be chosen such that

$$
D_{1}<r_{1}<D_{2}, r_{2}<S_{2},
$$

where $D_{1}$ is the maximal distance between neighboring texture details, $D_{2}$ is the minimal distance to isolated features, and $S_{2}$ is the minimal size of texture regions. Comparing Figs. 2(b) and (c) illustrates how $r_{2}$ controls the minimal size of texture regions to be detected. In addition, for the case $r_{2}<r_{1}$, isolated features of size $S_{1}$ are not suppressed if $r_{2}<S_{1}<r_{1}$. Therefore, choices of $r_{1}$ and $r_{2}$ with $r_{2}<S_{1}<r_{1}$ should be avoided.
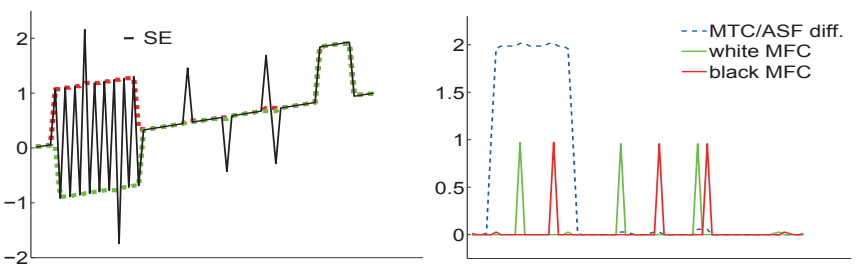

Fig. 1. Left: An artificial signal composed of a slowly varying component, a texture region, and individual features. Upper and lower envelopes of the texture obtained with alternating morphological filters are shown by red and green dashed lines. Right: Extraction of the texture region and individual features with the MTC and ASF diff. (Sec. 2) and the MFC (Sec. 4) operators. Note that MTC and ASF diff. yield identical responses for 1D signals (Proposition 4).

Alternatively, we introduce an operator defined as the difference between alternating sequential filters $\varphi \gamma \varphi$ and $\gamma \varphi \gamma$ with identical structuring elements

$$
\psi_{\mathrm{ASF}}(f)=\varphi \gamma \varphi(f)-\gamma \varphi \gamma(f)
$$

${ }^{3}$ The satellite image was logarithmically transformed before applying the MTC operator, see Sec. 3.1 for the rationale behind.
Alternating sequential filters $\varphi \gamma \varphi$ and $\gamma \varphi \gamma$ are ordered (Serra and Vincent, 1992; Soille, 2003), which ensures that ASF difference (ASF diff.) always yields non negative values, allowing to drop the $|.|^{+}$operator used in the MTC. In the next proposition we outline two properties of the ASF diff. that relate it to the MTC.

\section{Proposition 4. The MTC operator defined in Eq. (1)}

1. has lower response than the ASF diff. with the same SE, i.e. $\psi_{\mathrm{ASF}}(f) \geq \psi_{\mathrm{MTC}}(f)$.

2. is identical to the ASF diff. with the same SE in the $1 D$ case.

The first property holds in general and is directly followed from extensivity of closing and anti-extensivity of opening operators. The second property above follows from the fact that in the 1D case we have $\varphi \gamma \varphi=\gamma \varphi$ and $\gamma \varphi \gamma=\varphi \gamma$ (Serra and Vincent, 1992). The $\psi_{\mathrm{ASF}}$ operator applied to the artificial 1D signal is shown in the blue dashed line Fig. 1. As we expect it has the same response as the $\psi_{\mathrm{MFC}}$ operator. In the $2 \mathrm{D}$ case the ASF diff. and the MTC operators are not equivalent. However, we will show in Sec. 3 that they perform almost identically in their ability to distinguish texture from individual features.

An important property of the ASF diff. and the MTC operators is that they neither extend nor blur the borders of textured regions, thereby allowing accurate localization of texture borders. This property is illustrated in Fig. 3 in the rightmost column. Below we outline three other properties of the the ASF diff. and the MTC that are desirable for texture detection.

Proposition 5. The MTC operator in Eq. (3) and the ASF diff. (denoted in this proposition $\psi$ without subscript) are

1. bias invariant, $\psi(f)=\psi(f+a)$,

2. invariant to signal inversion ${ }^{4}, \psi(f)=\psi(a-f)$,

3. proportional to signal magnitude $\psi(a f)=|a| \psi(f)$,

where $a \in \mathbb{R}$ is a constant.

These properties follow from the general property of morphological operators with flat structuring elements to commute with increasing continuous functions ${ }^{5}$ and from the duality of opening and closing. Namely, if we denote by $\zeta$ opening or closing operators and by $\zeta^{*}$ their dual (opening for closing and closing for opening), bias invariance follows from $\zeta(f+a)=a+\zeta(f)$, invariance to signal inversion follows from $\zeta(a-f)=a-\zeta^{*}(f)$, and proportionality to signal magnitude follows from $\zeta(a f)=a \zeta(f), a \geq 0$ and $\zeta(a f)=a \zeta^{*}(f), a<0$. As an example we prove the second property for the MTC operator $\psi_{M T C}(f)$ defined in Eq. (3).

\footnotetext{
${ }^{4}$ In Mathematical Morphology signal inversion is also referred to as selfcomplementarity (Soille, 2003, 2005).

${ }^{5}$ In Mathematical Morphology increasing and continuous functions are usually termed anamorphoses (Serra, 1982).
} 


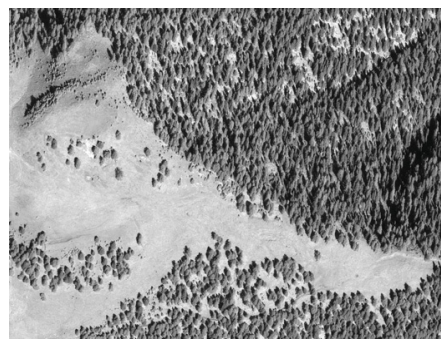

(a)

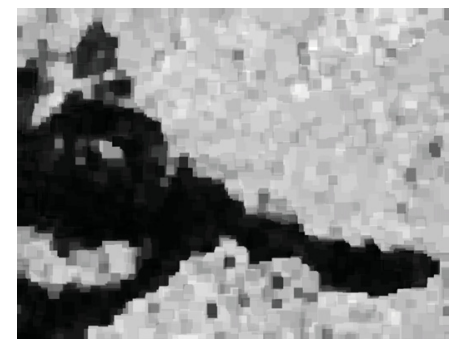

(b)

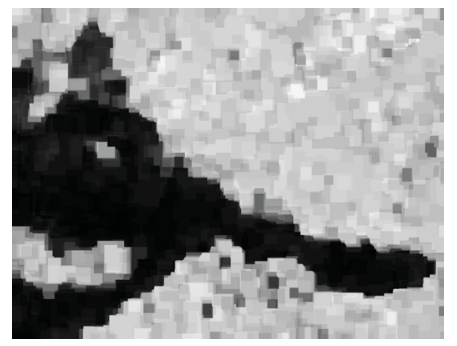

(c)



(d)

Fig. 2. (a) Pan-chromatic satellite image of 1500x1150 pixel size (C) GeoEye 2011, distributed by e-GEOS). (b) and (c) The MTC descriptor. $r_{1}=30, r_{2}=25$ in (b) and $r_{1}=30, r_{2}=35$ in (c). (d) Extraction of individual dark features, i.e. individual trees, using the $\psi_{\mathrm{MFC}}^{-}$operator with $r_{1}=r_{2}=30$ (Sec. 4). Note that the trees in forest areas are almost completely suppressed.

Proof.

$$
\begin{aligned}
\psi_{\mathrm{MTC}}(a-f) & =\left|\gamma_{r_{2}} \varphi_{r_{1}}(a-f)-\varphi_{r_{2}} \gamma_{r_{1}}(a-f)\right|^{+} \\
& =\left|\gamma_{r_{2}}\left(a-\gamma_{r_{1}}(f)\right)-\varphi_{r_{2}}\left(a-\varphi_{r_{1}}(f)\right)\right|^{+} \\
& =\left|a-\varphi_{r_{2}} \gamma_{r_{1}}(f)-\left(a-\gamma_{r_{2}} \varphi_{r_{1}}(f)\right)\right|^{+} \\
& =\left|\gamma_{r_{2}} \varphi_{r_{1}}(f)-\varphi_{r_{2}} \gamma_{r_{1}}(f)\right|^{+}=\psi_{\mathrm{MTC}}(f) .
\end{aligned}
$$

Although, the MTC was developed to discriminate texture and non-texture regions, its multi-scale extension can also be used for classification of different types of texture. The MTC computed for varying sizes of the SE generates a set of features that can be used for this purpose. Since accurate localization is an inherent property of the MTC, we expect that such MTCbased classification will be more accurate at texture borders in comparison to other texture classification approaches that involve the computation of summary statistics of dedicated features within a window, such as standard GLCM (Haralick et al., 1973) or a more recent approach based on linear contact distribution (Epifanio and Soille, 2007). In addition, for the MTCbased classification no preprocessing is required to mask out isolated structures (which are not part of any texture class) that may disturb classification results. The extension of the MTC approach to texture classification, as opposed to texture detection considered here, is, however, beyond the scope of this paper.

\section{Comparison of texture contrast descriptors}

In this section we compare the performance of the MTC operator with the ASF diff., the TER, the CC, the MaxMin difference, the StD, the LBP contrast, the AMF, and the PanTex algorithms. We denote by $w$ the scale parameter required for all algorithms. For the MTC operator it equals the size of the structuring elements $r_{1}=r_{2}$. To allow a consistent comparison, a few algorithms were slightly modified as follows.

In the $\mathrm{CC}$ algorithm we avoided several parameters suggested by the authors since they need to be adjusted for each type of image. Specifically, we used the simple product of contrast and number of connected components. Instead of disjoint blocks, a sliding window of the single size $w$ was used to compute the texture measure at each pixel as in the other compared methods. In the PanTex algorithm we used a square root of contrast measure derived from the GLCM matrix. This contrast measure can actually be computed without an explicit calculation of the GLCM matrix. The original PanTex index was designed with a single window of 9 pixels size, which was adjusted to $5 \mathrm{~m}$ satellite image resolution. Ten displacement vectors with the length varying from $w / 9$ to $w \sqrt{5} / 9$ were chosen in order to cover the full range of possible orientations. In our comparison we computed the GLCM contrast measure within a window of an arbitrary size $w$, such that displacement vectors of an approximate length $\frac{w}{7}$ were determined by all pixels on a discrete circle of radius $\frac{w}{7}$. Taking shorter displacement vectors did not significantly change the performance of PanTex, while taking longer vectors, as in Zingman et al. (2013b), reduced the performance estimated in Sec. 3.2.

To compute the LBP local variance and the AMF measures, we used a Matlab code available online ${ }^{6}$. In the LBP we used the square root of local variance computed as a variance of $4(w-1)$ equally spaced point samples on a circle of radius Round $\left(\frac{w}{2}\right)$. This, for example, gives 2, 4, 18 orientations (or the doubled number of directions), and radii 1, 2, 5 for $w$ equal to $2,3,10$, respectively. The AMF approach does not contain an analysis window, but contains a free parameter, which is the largest period of a sinusoid in the Gabor filters used in the AMF. We set this largest period of a sinusoid to $3 w$ pixels. In the quantitative comparison in Sec. $3.2 w$ varies from 10 to 70. The corresponding largest periods of the AMF approach included the recommended value given in the AMF code mentioned above. We also noted that choosing larger values only decreased the performance.

Note that similar to the MTC operator, all the algorithms, after the small modifications described above (except for AMF), fulfill the properties of Proposition 5.

\subsection{Qualitative comparison}

The texture contrast descriptors obtained using the compared transformations are shown in Fig. 3. The first two original images are of a size of $512 \times 512$ pixels; the third image is a panchromatic satellite image of $1300 \times 1100$ pixels size ${ }^{7}$; the fourth image is an enlarged part of the third image. $w=10$ for the first

\footnotetext{
${ }^{6}$ Matlab sources are available at http://www.cse.oulu.fi/CMV/Downloads/LBPMatlab and http://cvsp.cs.ntua.gr/software/texture/

${ }^{7}$ C GeoEye 2011, distributed by e-GEOS.
} 
two images and $w=30$ for the satellite images. For the case of satellite images, we applied all compared operators to logarithmically transformed images. Such preprocessing improves the robustness of the texture contrast descriptors to illumination variations (Zingman et al., 2012), and provides visual results of higher contrast. Given the limited space for this paper, we refrained from showing the MaxMin diff., LBP contrast and the AMF descriptors. Corresponding illustrations are, however, provided in Zingman et al. (2013b). The performance of the MTC operator and the ASF diff. is undistinguishable. All the descriptors have high values in textured areas and low values in smooth areas. However, contrary to the MTC operator and the ASF diff., the other approaches yield also high responses at isolated features that do not belong to texture. The TER operator is able to suppress step and ramp edges, but yields a high response at isolated ridges and blobs. The PanTex descriptor partially succeeds to suppress isolated curvilinear structures.

To better visualize the accuracy in texture localization, the texture descriptors were superimposed on the enlarged part of the satellite image in the fourth column of Fig. 3, where the contrast of red tones is proportional to the values of the descriptors. Since the distribution of descriptor values is strongly bimodal, one can distinguish two major levels of texture descriptors, low and high, that appear as a gray-reddish and saturated red overlaid on the original image. As can be seen from these images, another advantage of the MTC operator and the ASF diff. is that they do not extend the borders of texture regions as other methods do, except the TER. Our implementation of the $\mathrm{CC}$ method generates a halo near texture borders and around individual features. This effect does not occur in the original version of the CC method, in which disjoint/overlapping block processing was performed that, however, would not allow accurate texture localization.

\subsection{Quantitative comparison}

In order to quantitatively compare the ability of the methods to distinguish between texture and non-texture areas we quantify the separability between distributions of texture descriptors in these areas. We used the Fisher criterion (Fukunaga, 1990) that measures the distance between distribution means relative to their compactness. The criterion is given by $\frac{\left(\mu_{1}-\mu_{2}\right)^{2}}{\sigma_{1}^{2}+\sigma_{2}^{2}}$, where $\mu$ denotes the class mean and $\sigma^{2}$ denotes the class variance. Since ground truth data is required to define textured and non-texture regions, we created an artificial data set of gray-scale images along with corresponding masks of texture regions and nontexture areas, whereby the last also include individual features.

The data set consists of 100 images of $300 \times 300$ pixels with circular texture clusters and individual features (clusters may overlap; see the upper-left image in Fig. 5). The number of clusters and their diameters were chosen uniformly randomly and varied from 2 to 4 and from 60 to 120 pixels, respectively. The diameter of both individual features and texture details was 5 pixels. Texture details within clusters were placed at positions on a regular grid with random Gaussian offsets. The distance between nodes of the regular grid was set to 9 pixels. The amplitude of texture details and individual features varied randomly with normal distribution. White noise was added fol- lowed by smoothing with an averaging filter with a $3 \times 3$ kernel. The standard deviation of the noise was equal to one third of the amplitude of the texture details.

In the first two experiments, we set the mean amplitude of individual features equal and triple, respectively, of the amplitude mean of texture details. Figs. 4(a, b) show the resulting separability measure for the size parameter $w$ varying from 10 to 70 pixels. A comparison of these figures reveal a high degree of immunity of the MTC and ASF diff. to individual features even of high magnitude. In contrast, the performance of other methods severely decreased in the presence of such individual features.

In the third experiment we restricted the class of non-texture areas to smooth areas adjacent to texture regions and to individual features including their neighborhood. Fig. 4(c) shows the separability between such restricted non-texture areas and texture regions when mean amplitude of texture details and individual features is equal. A comparison of Fig. 4(c) and Fig. 4(a) confirms that the superiority in the performance of the MTC and ASF diff. methods stems from its ability to distinguish texture from isolated features as well as from smooth regions adjacent to texture borders. The disadvantage of the ASF diff. in comparison with the MTC is that the ASF diff. takes almost fifty percent more time to compute. In practice, this makes the MTC operator preferable for texture detection.

\section{Extraction of isolated features: The Morphological Fea- ture Contrast operator}

Using the ideas underlying the MTC operator, below we propose a Morphological Feature Contrast (MFC) operator that extracts isolated structures while suppressing texture details of textured background. Using alternating morphological filters, upper and lower texture envelopes were estimated in the MTC approach. To extract bright or dark individual features, we suggest using the difference between the original signal and one of its envelopes, as defined in the following equations

$$
\begin{aligned}
& \psi_{\mathrm{MFC}}^{+}(f)=\left|f-\gamma_{r_{2}} \varphi_{r_{1}}(f)\right|^{+}, \\
& \psi_{\mathrm{MFC}}^{-}(f)=\left|\varphi_{r_{2}} \gamma_{r_{1}}(f)-f\right|^{+} .
\end{aligned}
$$

To extract both types of individual features the sum of two operators should be used

$$
\psi_{\mathrm{MFC}}=\psi_{\mathrm{MFC}}^{+}+\psi_{\mathrm{MFC}}^{-} .
$$

We call $\psi_{\mathrm{MFC}}^{+}$and $\psi_{\mathrm{MFC}}^{-}$white and black MFC, respectively. The MFC operators applied to a 1D artificial signal are illustrated in Fig. 1. The appropriate sizes $r_{1}, r_{2}$ of the SEs should be chosen such that

$$
D_{1}<r_{1}<D_{2}, S_{1}<r_{2}<S_{2},
$$

where $D_{1}$ is the maximal distance between neighboring texture details, $D_{2}$ is the minimal distance to isolated features, $S_{1}$ is the maximal size of isolated features, and $S_{2}$ is the minimal size of texture regions. For the MFC operators these constraints ensure detection of isolated features and suppression of texture. As stated in the following proposition, the MFC operator $\psi_{\mathrm{MFC}}$ shares with the MTC the three properties defined in Sec. 2. 

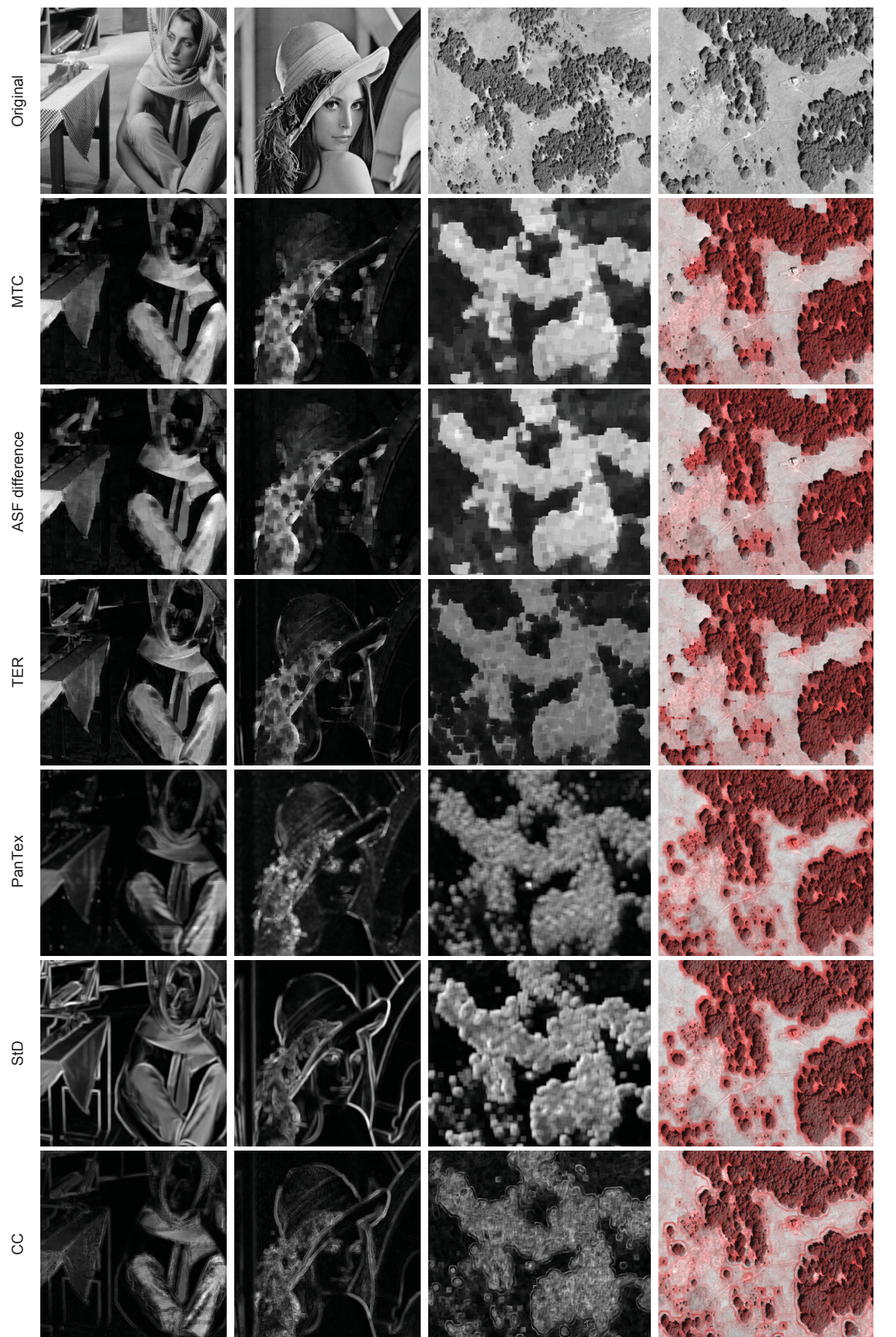

Fig. 3. Comparison of texture contrast descriptors. Details can be found in Sec. 3 and Sec. 3.1. 


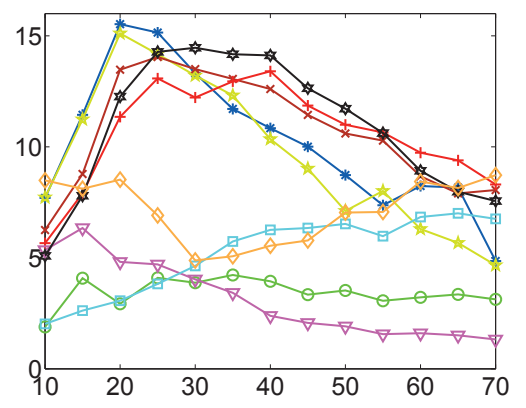

(a)

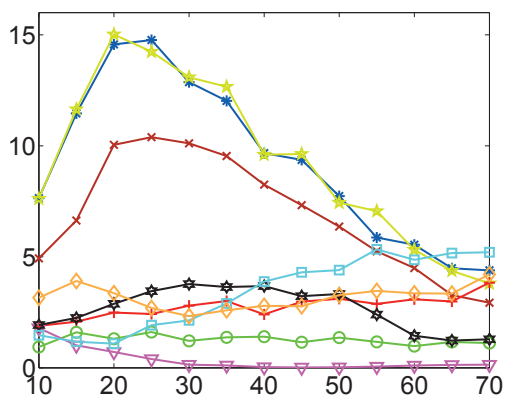

(b)

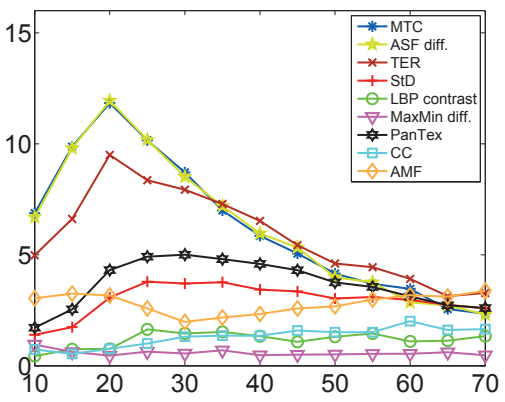

(c)

Fig. 4. (a) and (b): The measure of separability between texture and non-texture regions as a function of the scale parameter $w$. The mean amplitude of individual features is equal to the amplitude of texture details in (a) and tripled in (b). (c) The measure of separability of texture regions from areas around individual features and smooth areas adjacent to texture borders. The mean amplitude of individual features equals the amplitude of texture details.

Proposition 6. The $\psi_{\mathrm{MFC}}$ operator in Eq. (8) fulfills the three properties of Proposition 5, i.e it is bias invariant, invariant to signal inversion, and proportional to signal magnitude.

It can be shown that $\psi_{\mathrm{MFC}}$ is equal to $\max (\varphi \gamma(f), f)-$ $\min (\gamma \varphi(f), f)$, while $\psi_{\mathrm{MFC}}^{+}$and $\psi_{\mathrm{MFC}}^{-}$are equal to operators that were already defined in Salembier (1990) as $f-\min (\gamma \varphi(f), f)$ and $\max (\varphi \gamma(f), f)-f$, and were used for detection of defects in the noisy background of a metallic surface.

Fig. 5 and Fig. 2(d) show examples of the MFC operators $\psi_{\mathrm{MFC}}$ and $\psi_{\mathrm{MFC}}^{-}$applied to gray-scale images. Note that in all illustrations throughout this paper, dark tones represent high values of transformations extracting isolated features. One can observe that various individual features/objects were highlighted while texture areas were simultaneously suppressed. For example, in the right image in Fig. 5 the forest texture area and the texture of the village were suppressed, while isolated buildings (mostly bright roofs) outside the dense village center and isolated trees (mostly shadows of trees) were preserved in the output image. Additional examples can be found in Zingman et al. (2013b). The MFC operator is capable of suppressing texture areas even if composed of details of higher magnitude and similar shape in relation with the magnitude and shape of individual features. Although, several methods were developed to extract object boundaries (edge features) from textured background, we are not aware of other techniques that perform qualitatively similar to the MFC when extracting blob-like features (as well as features of arbitrary shape).

We have shown that the ASF diff., the MTC and the MFC operators have similar properties and are good in distinguishing texture from isolated features. The first two are complementary to the MFC operator in the sense that they respond to texture while the MFC responds to individual features. The qualitative behavior of these operators is briefly summarized in Table 1, which is conveniently interpreted along with Fig. 1. Note also that these operators do not respond to step edges and respond correctly (with 'Low') at smooth regions nearby texture borders and in the vicinity of isolated features.

\subsection{MFC based extraction of isolated linear features}

Above, we have shown that the MFC operators are capable of extracting features of different types with width smaller than
Table 1. Qualitative behavior of the MTC and ASF diff. versus the MFC operators.

\begin{tabular}{|c|cccc|}
\hline & Texture & $\begin{array}{c}\text { Isolated } \\
\text { features }\end{array}$ & $\begin{array}{c}\text { Isol. features } \\
\text { within texture }\end{array}$ & $\begin{array}{c}\text { Smooth } \\
\text { regions }\end{array}$ \\
\hline $\begin{array}{c}\text { MTC \& ASF } \\
\text { diff. } \\
\text { MFC }\end{array}$ & High & Low & High & Low \\
\hline
\end{tabular}

$r_{2}$. Features of a specific type can be extracted by a sequence of standard morphological transformations, with the structuring element shaped similarly to features. Here we illustrate advantages of the use of the MFC within such a sequence for the case of linear features.

The remotely sensed images in Fig. 6 (left) contain rectangular structures composed of nearly linear walls that were used as livestock enclosures. This type of structures is needed to be detected within the archaeological project (Lambers and Zingman, 2012, in press; Zingman et al., 2013a). The white top-hat transform is commonly used to remove background and emphasize small bright structures in images. Fig. 6 (the second column) shows the white top-hat transform followed by a filter $\gamma_{\text {lin }}$ obtained by the point-wise maximum of morphological openings with linear SE at 12 different orientations ranging from 0 to $\pi$. This sequence of morphological operators highlights narrow linear features longer than the length of the linear structuring element. However, texture details are also emphasized. Furthermore, an appropriate threshold setting is required to obtain a binary map of features.

To remove texture while keeping isolated features, the MFC operator $\psi_{\mathrm{MFC}}^{+}$can be applied prior to $\gamma_{\mathrm{lin}}{ }^{8}$. We will call the sequence of the MFC operator and $\gamma_{\text {lin }}$ the MFC based detector of linear features. White top-hat followed by the MFC based detector of linear features thresholded at zero level yields a map of linear features with most texture details removed, see an example in Fig. 6 (the third column). We used a 5x5 SE in the top-hat operator, $r_{1}=5, r_{2}=10$ in $\psi_{\mathrm{MFC}}^{+}$operator, and 15 pixels length for the linear SE in $\gamma_{\text {lin }}$. Note that threshold tuning was not required to obtain the result. For the case of linear

\footnotetext{
${ }^{8} \psi_{\mathrm{MFC}}^{+}$could also be directly applied to the initial image without the use of the white top-hat.
} 

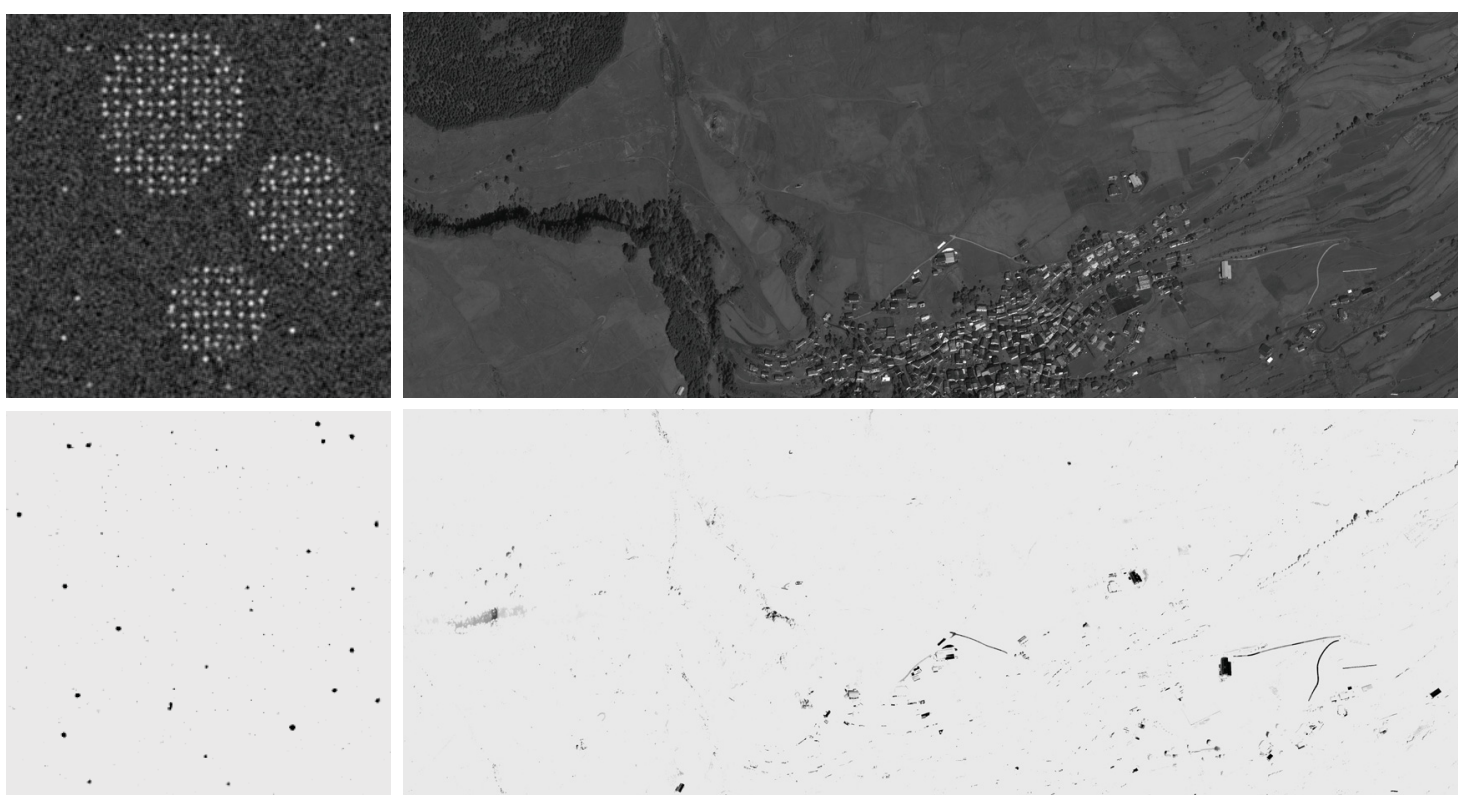

Fig. 5. First row: $312 \times 312$ artificial and 5200x1900 satellite (C) GeoEye 2011, distributed by e-GEOS) images. Second row: individual features extracted by means of the MFC operator $\psi_{\mathrm{MFC}} \cdot r_{1}=30, r_{2}=10$ for the artificial image and $r_{1}=r_{2}=90$ pixels for the satellite image.

(elongated) step edges, this approach can easily be adapted by replacing the top-hat transform with the morphological gradient (Rivest et al., 1993). To speed up the detector described above, openings with linear SEs can be implemented using techniques proposed in Soille and Talbot (2001).

\subsubsection{Comparison of the MFC based and the non-CRF inhibi- tion approaches for extraction of linear features}

One of the advantages of the MFC based detector of linear features is that it involves easily tunable geometry related parameters, i.e. $r_{1}, r_{2}$ for the MFC operator, and the length of the structuring element for linear openings $\gamma_{\text {lin }}$. These parameters define spatial constraints on the objects to be detected and texture to be suppressed. Moreover, final thresholding can always be performed at zero level, which results in robust detection of linear features in variable scenes and illumination conditions. In contrast, other methods usually involve parameters related to intensities of features or frequency of their appearance. For example, an efficient approach was recently developed for detection of object contours in cluttered scenes by means of biologically motivated non-classical receptive field (non-CRF) inhibition (Grigorescu et al., 2003). In this approach an inhibition level needs to be carefully tuned. The suitable value of this parameter may vary for different images depending on illumination conditions and ratio of features strength over clutter or texture. A multilevel inhibition technique was suggested in $\mathrm{Pa}-$ pari and Petkov (2011) to address this problem. It makes the approach more robust, however, it may reduce the performance when applied to a particular image comparing to the single optimal inhibition level. In addition, a multilevel inhibition involves a fraction constant $p$ with an appropriate value depending on the size and the number of structures of interest in the image.

Below, we compare the MFC based and the non-CRF based detectors of linear features. In this comparison we adapted the isotropic non-CRF inhibition approach (Grigorescu et al., 2003) to extraction of linear ridge (bar) features by using only an even filter in the Gabor energy. Note, that the Gabor energy is not invariant to constant bias because an even Gabor filter is not a zero-DC filter. The right-most column in Fig. 6 shows the result of applying the non-CRF inhibition approach with the parameters chosen for the best visual results, keeping the structures of our interest extracted with the lowest level of clutter. In our example the optimal inhibition level is $\alpha=1.8$. For the Gabor filter the standard deviation is $\sigma=2$, the spatial aspect ratio $\gamma=0.25$, and the wavelength $\lambda=5.7$. For the inhibition term the standard deviation is $\sigma=1.5$. For visualization purposes the output of the non-CRF inhibition method was logarithmically transformed. Additional comparative examples of the MFC based and the non-CRF based detectors can be found in Zingman et al. (2013b).

To quantitatively compare the MFC based and the non-CRF based detectors we generated a set of $26512 \times 512$ artificial images composed of Brodatz textures and patterns of linear features. Ground truth linear features were created as dashed lines with an amplitude equal 0.8 , three pixels width and 20 pixels segment length. They were blurred by convolution with a $3 \times 3$ averaging kernel and then added to textures (see Fig. 7 on the left). The gap between line segments was equal to the length of segments. Line orientation was different for each texture and taken from all possible equally spaced orientations. Prior to the composition, the Brodatz textures were normalized to a unit standard deviation and multiplied by a linear intensity gradient image such that the right texture border was brighter than the left border by four times (see Fig. 7 on the left).

Since we have very unbalanced classes (the class of linear features is much smaller in size than the size of texture background), we used receiver operating characteristic (ROC) 

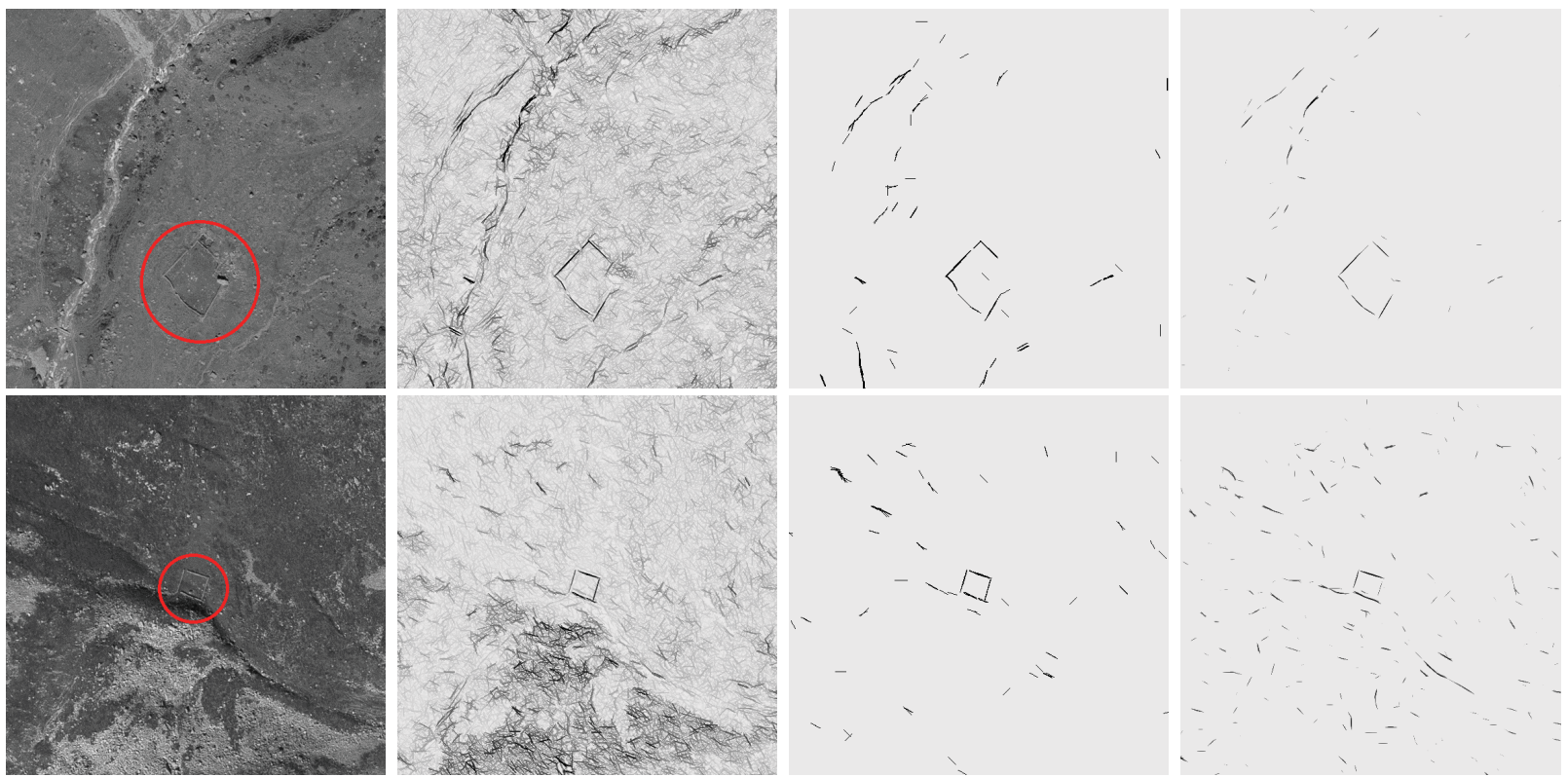

Fig. 6. First column: Aerial SWISSTOPO images (red channel) of 600x600 pixel size with man-made structures composed of linear walls. Second column: White top-hat transform followed by an opening filter $\gamma_{\text {lin }}$ obtained by the point-wise maximum of morphological openings with linear structuring elements at different orientations. Third column: White top-hat transform followed by the MFC based detector of isolated linear features ( $\psi_{\mathrm{MFC}}^{+}$with subsequent $\left.\gamma_{\text {lin }}\right)$. Non-zero pixels are shown in black. Fourth column: Non-CRF based detector of isolated linear features.

curves (Fawcett, 2006) to compare the detection performance of the algorithms. ROC curves were previously used for comparison of edge detectors in Bowyer et al. (2001), which also needed to account for unbalanced classes. A receiver operating characteristic (ROC) curve shows the relationship between true positives detection rate and false positives rate of a particular detector. The true positives rate is the relative number of pixels of linear features that were correctly identified, while the false positive rate is the relative number of pixels of texture background that were wrongly detected as linear features. An important advantage of ROC curves is that they summarize the performance of detectors for different class priors and detection error costs. Fig. 8 shows the resulting ROC curves for low values of false positives, where the MFC based detector is superior. For high false positives the non-CRF approach yields a higher true positives rate. Moreover, the ROC curve of the MFC based detector cannot be generated for true positives rates higher than shown in the figure, because this detector completely removes parts of linear features. This behavior fits visual results in Fig. 7 obtained for a couple of particular images (shown in Fig. 7 on the left) from the image dataset. For visual purposes, the output of the MFC and non-CRF based detectors was logarithmically transformed. It can be seen that the MFC based approach is more successful in suppressing texture background, but thins linear features.

ROC curves were generated with optimal detector parameters. In our experiments such parameters for the MFC based detector were $r_{1}=10, r_{2}=5$. The length of the linear SE for openings $\gamma_{\text {lin }}$ was equal 5. For the non-CRF based detector optimal parameters were $\sigma=3.6, \gamma=0.9, \lambda=10.3$ for the Gabor filter and $\sigma=3$ for the inhibition term. The inhibition level was $\alpha=1.8$.
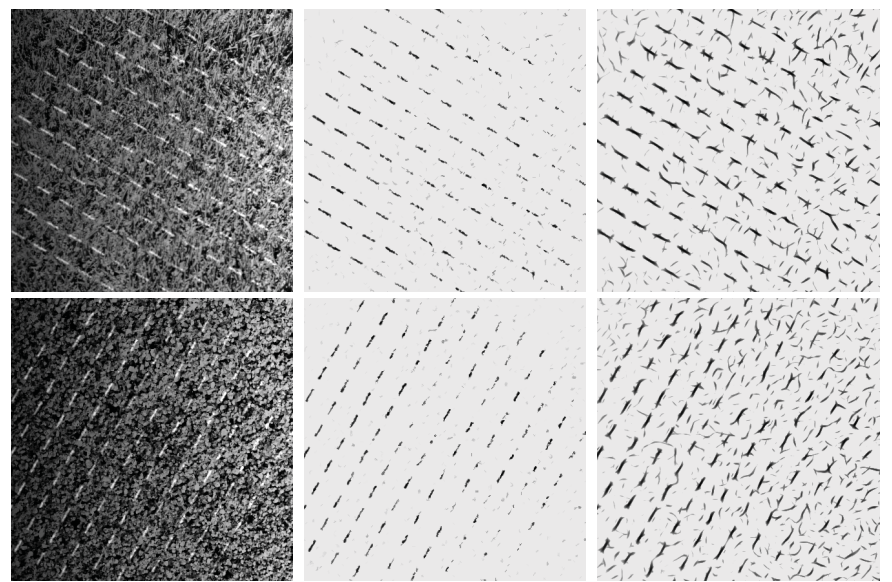

Fig. 7. Left: Examples of artificial images used for quantitative comparison of the MFC and the non-CRF based detectors of linear features. The images were composed of Brodatz textures and patterns of linear features (see details in Sec. 4.1.1). Middle: MFC based detector. Right: Non-CRF based detector.

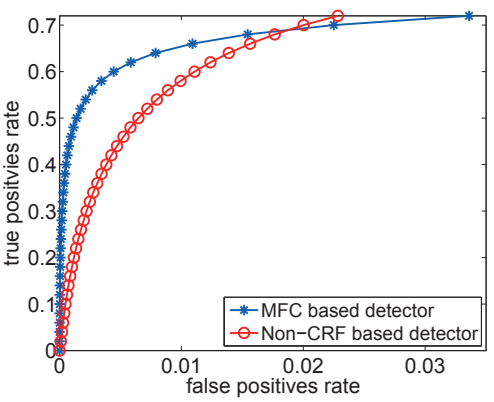

Fig. 8. ROC curves of the MFC and non-CRF based detectors of linear features. 


\subsection{Extension of the MFC operator to vector-valued images}

In this section, we generalize the MFC operators from grayscale to vector-valued discrete images, where each pixel is attributed by a vector of values. A multispectral image is an example of such a vector-valued image. The problem with extending morphological operators to vector-valued images lies in the lack of a natural ordering of vectors. However, some morphological transformations defined in terms of arithmetic differences between morphological operators can naturally be extended to vector-valued images without the need of chosing a vectorial order. Examples of such extended transformations were recently proposed for morphological gradient and for tophat in Evans and Liu (2006) and Hanbury (2004). Using similar ideas, we derive an extended version of the MFC operators below.

Proposition 7. The MFC operators $\psi_{\mathrm{MFC}}^{-}(f), \psi_{\mathrm{MFC}}^{+}(f)$, defined in Eq. (7) and in Eq. (6) with structuring elements of sizes $r_{1}$ and $r_{2}$, can be expressed in the following forms

$$
\begin{aligned}
& {\left[\psi_{\mathrm{MFC}}^{-}(f)\right](x)=\min _{k \in B_{x}^{(2)}} \max _{j \in B_{k}^{(3)}} \min _{i \in B_{j}^{(1)}}|f(i)-f(x)|^{+},} \\
& {\left[\psi_{\mathrm{MFC}}^{+}(f)\right](x)=\min _{k \in B_{x}^{(2)}} \max _{j \in B_{k}^{(3)}} \min _{i \in B_{j}^{(1)}}|f(x)-f(i)|^{+},}
\end{aligned}
$$

where $B_{p}^{(1)}$ and $B_{p}^{(2)}$ are structuring elements of of sizes $r_{1}$ and $r_{2}$, respectively, shifted to $p$, and $B^{(3)}$ denotes the structuring element $B^{(1)}$ dilated by $B^{(2)}$.

Proof. Let us denote by $\delta$ and $\varepsilon$ morphological dilation and erosion, respectively. The MFC operator defined in Eq. (7) can be rewritten as follows

$$
\begin{aligned}
& {\left[\psi_{\mathrm{MFC}}^{-}(f)\right](x)=\left|\left[\varepsilon_{r_{2}} \delta_{r_{2}} \delta_{r_{1}} \varepsilon_{r_{1}}(f)\right](x)-f(x)\right|^{+}} \\
& =\left|\left[\min _{k \in B_{x}^{(2)}} \max _{j \in B_{k}^{(3)}} \min _{i \in B_{j}^{(1)}} f(i)\right]-f(x)\right|^{+}=\left|\min _{k \in B_{x}^{(2)}}\left(\left[\max _{j \in B_{k}^{(3)}} \min _{i \in B_{j}^{(1)}} f(i)\right]-f(x)\right)\right|^{+}
\end{aligned}
$$

We note that for an arbitrary function $g(y)$

$$
\begin{aligned}
& \left|\min _{y \in B_{x}}(g(y)-f(x))\right|^{+} \\
& = \begin{cases}\min _{y \in B_{x}}(g(y)-f(x)), & \text { if } \forall y \in B_{x}: g(y) \geq f(x) \\
0, & \text { otherwise }\end{cases} \\
& =\min _{y \in B_{x}}|g(y)-f(x)|^{+},
\end{aligned}
$$

and

$$
\begin{aligned}
& \left|\max _{y \in B_{x}}(g(y)-f(x))\right|^{+} \\
& = \begin{cases}\max _{y \in B_{x}}(g(y)-f(x)), & \text { if } \exists y \in B_{x}: g(y) \geq f(x) \\
0, & \text { otherwise }\end{cases} \\
& =\max _{y \in B_{x}}|g(y)-f(x)|^{+} .
\end{aligned}
$$

Thus, we can proceed with

$$
\begin{aligned}
& \left|\min _{k \in B_{x}^{(2)}}\left(\left[\max _{j \in B_{k}^{(3)}} \min _{i \in B_{j}^{(1)}} f(i)\right]-f(x)\right)\right|^{+}=\min _{k \in B_{x}^{(2)}}\left|\left[\max _{j \in B_{k}^{(3)}} \min _{i \in B_{j}^{(1)}} f(i)\right]-f(x)\right|^{+} \\
& =\min _{k \in B_{x}^{(2)}}\left|\max _{j \in B_{k}^{(3)}}\left(\left[\min _{i \in B_{j}^{(1)}} f(i)\right]-f(x)\right)\right|^{+}=\min _{k \in B_{x}^{(2)}} \max _{j \in B_{k}^{(3)}}\left|\left[\min _{i \in B_{j}^{(1)}} f(i)\right]-f(x)\right|^{+} \\
& =\min _{k \in B_{x}^{(2)}} \max _{j \in B_{k}^{(3)}}\left|\min _{i \in B_{j}^{(1)}}(f(i)-f(x))\right|^{+}=\min _{k \in B_{x}^{(2)}} \max _{j \in B_{k}^{(3)}} \min _{i \in B_{j}^{(1)}}|f(i)-f(x)|^{+} .
\end{aligned}
$$

This proves Eq. (10). Eq. (11) can be proved similarly.

We now define a new vectorial MFC operator $\psi_{\mathrm{MFC}}(\bar{f})$ that applies to vector-valued images $\bar{f}$. We replace the non-negative difference between intensity values in Eq. (10) and Eq. (11) by a suitable metric distance $D$ between vectors,

$$
\left[\psi_{\mathrm{MFC}}(\bar{f})\right](x)=\min _{k \in B_{x}^{(2)}} \max _{j \in B_{k}^{(3)}} \min _{i \in B_{j}^{(1)}} D(\bar{f}(x), \bar{f}(i))
$$

In contrast to $\psi_{\mathrm{MFC}}^{+}(f)$ and $\psi_{\mathrm{MFC}}^{-}(f)$, the $\psi_{\mathrm{MFC}}(\bar{f})$ operator extracts both dark and bright structures when applied to multispectral images. If one is interested in extracting either dark (or bright) structures only, i.e. structures having low (or high) values relative to background, in all channels, pseudo-distances may be used. For example, instead of using the $D_{\infty}$ distance, pseudo distances defined by $D_{\infty}^{+}(\bar{f}, \bar{g})=\max _{i}\left|f_{i}-g_{i}\right|^{+}$and $D_{\infty}^{-}(\bar{f}, \bar{g})=\max _{i}\left|g_{i}-f_{i}\right|^{+}$may be employed.

Vectorial operators may or may not be preferable to an independent processing of channels of a vector-valued image followed by integration of the results. Another alternative is to transform a vector valued image to a single channel image before processing. Finding a proper transformation or a way to combine independently processed channels is a task dependent problem, often approached by trial and error. A similar problem appears in the case of vectorial MFC, because a suitable distance must be chosen. Fig. 9 shows examples of vectorial MFC with Euclidean $D_{2}(\bar{f}, \bar{g})=\|\bar{f}-\bar{g}\|_{2}$ and angular (spectral) distances $D_{\alpha}(\bar{f}, \bar{g})=(\bar{f} \cdot \bar{g}) /\left(\|\bar{f}\|_{2}\|\bar{g}\|_{2}\right)$. A comparative evaluation of the vectorial MFCs, however, is beyond the scope of this paper.

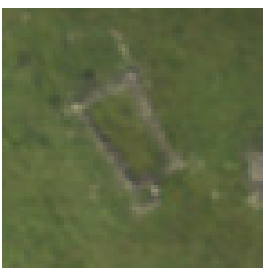

(a)

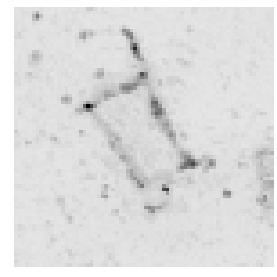

(b)

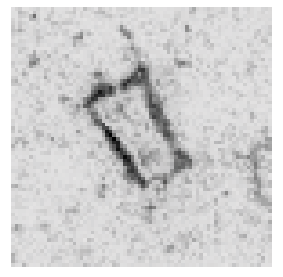

(c)
Fig. 9. An example of application of the vectorial MFC operator to an aerial RGB (SWISSTOPO) image patch with an architectural structure shown in (a). Vectorial MFC with (b) Euclidian distance and (c) angular distance.

\section{Conclusions}

We have shown how alternating morphological filters can be used to design operators for detection of texture regions and isolated features. The morphological texture contrast (MTC) operator is designed for various applications where high contrast textures of different types should be segmented out from nontexture regions that may also contain isolated features. This operator is able to discriminate texture from isolated features irrespectively of their high magnitude and has a good localization property. The comparison of the MTC operator based on visual inspection and quantitative experiments, revealed its superiority over other methods used for texture detection. 
The morphological feature contrast (MFC) operator was proposed for extraction of isolated features while simultaneously being able to suppress texture background details. It has a similar underlying structure as the MTC operator. An extension of the MFC operator was derived allowing the MFC operator to be applied directly to vector-valued images. A simple scheme based on the MFC operator was designed for detection of isolated linear features. The advantages of this detector over the recently introduced non-classical receptive field inhibition approach were discussed and evaluated. We conclude that the MTC and the MFC operators excel in tasks in which it is important to distinguish isolated features from texture details.

\section{Acknowledgments}

This work was funded by the Interreg IV Program "Alpenrhein - Bodensee - Hochrhein" and by the Zukunftskolleg, University of Konstanz. It was also supported by the DFG Research Training Group GK-1042 "Explorative Analysis and Visualization of Large Information Spaces." We also thank the anonymous reviewers, who helped to improve the quality of the paper.

\section{References}

Bergman, R., Nachlieli, H., Ruckenstein, G., 2008. Detection of textured areas in natural images using an indicator based on component counts. J. Electronic Imaging 17, 043003-1-043003-13.

Bowyer, K.W., Kranenburg, C., Dougherty, S., 2001. Edge detector evaluation using empirical ROC curves. Computer Vision and Image Understanding 84, 77-103.

Dinstein, I., Fong, A., Ni, L., Wong, K., 1984. Fast discrimination between homogeneous and textured regions, in: Proc. of Int. Conf. on Pattern Recognition, Montreal, Canada. pp. 361-363.

Dubuc, B., Zucker, S., 2001. Complexity, confusion, and perceptual grouping. Part II: Mapping complexity. J. Mathematical Imaging and Vision 15, 83116.

Epifanio, I., Soille, P., 2007. Morphological texture features for unsupervised and supervised segmentations of natural landscapes. IEEE Transactions on Geoscience and Remote Sensing 45, 1074-1083.

Evans, A.N., Liu, X.U., 2006. A morphological gradient approach to color edge detection. IEEE Trans. Image Processing 15, 1454-1463.

Fawcett, T., 2006. An introduction to ROC analysis. Pattern Recogn. Lett. 27, 861-874.

Fukunaga, K., 1990. Introduction to Statistical Pattern Recognition. Academic Press.

Gonzalez, R.C., Woods, R.E., 2002. Digital Image Processing.

Grigorescu, C., Petkov, N., Westenberg, M., 2003. Contour detection based on non-classical receptive field inhibition. IEEE Trans. Image Processing. 12, 729-739.

Hanbury, A., 2004. The morphological top-hat operator generalised to multichannel images, in: Proc. of the Int. Conf. on Pattern Recognition, pp. 672675.

Haralick, R.M., Shanmugam, K., Dinstein, I., 1973. Textural features for image classification. IEEE Trans. Systems Man and Cybernetics 6, 610-621.

Karu, K., Jain, A.K., Bolle, R.M., 1996. Is there any texture in the image? Pattern Recognition 29, 1437-1446.

Kokkinos, I., Evangelopoulos, G., Maragos, P., 2009. Texture analysis and segmentation using modulation features, generative models and weighted curve evolution. IEEE Trans. Pattern Analyis and Machine Intelligence 31, $142-157$.

Lambers, K., Zingman, I., 2012, in press. Towards detection of archaeological objects in high-resolution remotely sensed images: the Silvretta case study, in: Proc. of Computer Appl. and Quantitative Methods in Archaeology, Southampton, UK.
Ojala, T., Pietikäinen, M., Mäenpää, T., 2002. Multiresolution gray-scale and rotation invariant texture classification with local binary patterns. IEEE Trans. Pattern Anal. Mach. Intell. 24, 971-987.

Papari, G., Petkov, N., 2011. An improved model for surround suppression by steerable filters and multilevel inhibition with application to contour detection. Pattern Recognition 44, 1999 - 2007.

Pesaresi, M., Gerhardinger, A., Kayitakire, F., 2008. A robust built-up area presence index by anisitropic rotation-invariant textural measure. IEEE Journal of selected topics in applied earth observation an remote sensing 1, 180192.

Rivest, J.F., Soille, P., Beucher, S., 1993. Morphological gradients. J. Electronic Imaging 2, 326-336.

Salembier, P., 1990. Comparison of some morphological segmentation algorithms based on contrast enhancement - application to automatic defect detection, in: European Signal Processing Conference, Barcelona, Spain. pp. $833-836$.

Serra, J., 1982. Image Analysis and Mathematical Morphology. Academic Press.

Serra, J., Vincent, L., 1992. An overview of morphological filtering. Circuits, Systems, and Signal Processing 11, 47-108.

Soille, P., 2003. Morphological Image Analysis: Principles and Applications. 2 ed., Springer-Verlag Berlin.

Soille, P., 2005. Beyond self-duality in morphological image analysis. Image and Vision Computing 23, 249-257.

Soille, P., Talbot, H., 2001. Directional morphological filtering. IEEE Transactions on Pattern Analysis and Machine Intelligence 23, 1313-1329.

Verbeek, P.W., Vrooman, H.A., Vliet, L.J.V., 1988. Low-level image processing by max-min filters. Signal Processing 15, 249-258.

Zingman, I., Saupe, D., Lambers, K., 2012. Morphological operators for segmentation of high contrast textured regions in remotely sensed imagery, in: Proc. of the IEEE Int. Geoscience and Remote Sensing Symposium, Munich, Germany. pp. 3451-3454.

Zingman, I., Saupe, D., Lambers, K., 2013a. Automated search for livestock enclosures of rectangular shape in remotely sensed imagery, in: Proc. SPIE, Image and Signal Processing for Remote Sensing.

Zingman, I., Saupe, D., Lambers, K., 2013b. Detection of texture and isolated features using alternating morphological filters, in: Hendriks, C., Borgefors, G., Strand, R. (Eds.), Mathematical Morphology and Its Applications to Signal and Image Processing. Springer. volume 7883 of Lecture Notes in Computer Science, pp. 440-451. 